\title{
Evaluation of the use of long noncoding RNAs as biomarkers for diagnosis of breast cancer
}

\author{
Nahla El-Ashmawy ${ }^{1}$, Fatma Zakaria ${ }^{2}$, Ghada Al-Ashmawy ${ }^{1}$ and Sara Hamouda ${ }^{1}$ \\ ${ }^{1}$ Department of Biochemistry, Faculty of Pharmacy, Tanta University, Tanta, Egypt \\ 2 Oncology Department, Faculty of Medicine, Tanta University, Tanta, Egypt
}

Background: FAM83H antisense RNA 1 (FAM83H-AS1) and long noncoding RNA activated by TGF $\beta$ (IncRNA-ATB) are two IncRNAs that have tumor promoting functions in breast cancer (BC). Matrix metalloproteinase-9 (MMP-9) is another tumor marker that exerts an oncogenic role in $B C$ and facilitate cancer invasion and metastasis. Aims: Our study aimed to 1) analyze serum levels of FAM83HAS1, IncRNA-ATB, and MMP-9 in BC patients, 2) compare their diagnostic role with that of CA15-3 in $\mathrm{BC}$ patients with different stages (I-II, III, and IV), and 3) correlate the levels of the measured IncRNAs, MMP-9, and CA15-3 with the clinicopathological features of BC. Materials and Methods: Serum FAM83H-AS1 and IncRNA-ATB levels were analyzed in $90 \mathrm{BC}$ patients and 30 healthy controls using RTPCR. While serum levels of MMP-9 and CA15-3 were measured in the same studied groups by ELISA. Results: FAM83H-AS1, IncRNA-ATB, MMP-9, and CA15-3 levels were significantly elevated in sera of BC patients. ROC curve analysis showed that IncRNA-ATB and MMP-9 had higher AUC values than CA15-3 in diagnosis of stage I-II patients (AUC: 0.844 and 0.898, $p=0.000$ for IncRNA-ATB and MMP-9, respectively versus $0.738, p=0.002$ for CA15-3). FAM83H-AS1 level was significantly correlated with higher stage, larger tumor size, positive lymph node metastasis, and distant metastasis. FAM83H-AS1 level was also significantly increased in PR-negative and ki67-rich tumors. Conclusion: Our study demonstrated that circulating IncRNA-ATB and MMP-9 could be used as diagnostic biomarkers for early BC. FAM83H-AS1 could also be a potential candidate for staging BC and monitoring disease progression.

Keywords: Breast cancer diagnosis; FAM83H-AS1; LncRNA-ATB; Long non-coding RNA; MMP-9

Editor-in-Chief: Prof. M.L. Salem, PhD - Article DOI: 10.21608/jcbr.2021.60072.1153 